\title{
ERGODIC POTENTIALS WITH A DISCONTINUOUS SAMPLING FUNCTION ARE NON-DETERMINISTIC
}

\author{
DAVID DAMANIK AND ROWAN KILLIP
}

\begin{abstract}
We prove absence of absolutely continuous spectrum for discrete onedimensional Schrödinger operators on the whole line with certain ergodic potentials, $V_{\omega}(n)=f\left(T^{n}(\omega)\right)$, where $T$ is an ergodic transformation acting on a space $\Omega$ and $f: \Omega \rightarrow \mathbb{R}$. The key hypothesis, however, is that $f$ is discontinuous. In particular, we are able to settle a conjecture of Aubry and Jitomirskaya-Mandel'shtam regarding potentials generated by irrational rotations on the torus.

The proof relies on a theorem of Kotani, which shows that non-deterministic potentials give rise to operators that have no absolutely continuous spectrum.
\end{abstract}

\section{Introduction}

Consider the discrete quasi-periodic Schrödinger operator

$$
\left[H_{\omega} \phi\right](n)=\phi(n+1)+\phi(n-1)+\lambda f(n \alpha+\omega \quad \bmod 1) \phi(n)
$$

on $\ell^{2}(\mathbb{Z})$ where $\lambda>0$ denotes the coupling constant, $f: \mathbb{R} / \mathbb{Z} \rightarrow \mathbb{R}$ is bounded, $\alpha$ is irrational, and $\omega \in[0,1)$.

When $f$ is continuous, the existence of absolutely continuous spectrum is a difficult question that has attracted a great deal of attention recently. In this note, we will show that for discontinuous $f$, there is none.

The absence of absolutely continuous spectrum for such $f$ was conjectured by Aubry [1] and by Jitomirskaya and Mandel'shtam [14].

Theorem 1. If $f$ has a single (non-removable) discontinuity, then for all $\omega \in$ $[0,1)$, the operator $H_{\omega}$ has no absolutely continuous spectrum.

We will actually prove a far more general result; see Theorem 2. Irrational rotations on $\mathbb{R} / \mathbb{Z}$ can be replaced by a very general dynamical system and the restrictions on $f$ can be significantly relaxed.

In addition to addressing the conjecture described above, this particular example makes a link to some recent research, which we will now describe.

If $f$ is nice enough, one expects purely absolutely continuous spectrum for small $|\lambda|$ and purely point spectrum (with exponentially decaying eigenfunctions) when $|\lambda|$ is large. (The latter scenario is usually referred to as Anderson localization.) Matters are particularly well understood when $f(x)=\cos (2 \pi x)$; see Jitomirskaya [7] and the references therein. In this case, the operator $H$ has

Received February 27, 2004.

D. D. was supported in part by NSF grant DMS-0227289. 
purely absolutely continuous spectrum (for almost all $\alpha$ and $\omega$ ) when $|\lambda|<2$ and localization occurs for $|\lambda|>2$ (again for almost all $\alpha$ and $\omega$ ).

Under analyticity assumptions on $f$, localization was shown by Bourgain and Goldstein, $[3,4]$, for $|\lambda|$ large enough; while for sufficiently small $\lambda$, Bourgain and Jitomirskaya, $[3,5]$, proved purely absolutely continuous spectrum.

It is desirable to prove these results under weaker regularity assumptions on $f$ (see [2] and [8] for recent developments in this direction) and, at the same time, explore the breakdown of these results once $f$ becomes too singular.

It is known that these results definitely do break down when $f$ takes only finitely many values. In this case, absence of localization in almost all cases was shown by Delyon and Petritis in [6] and absence of absolutely continuous spectrum in all cases is a result of Kotani [11]. Notice that these results hold for all values of the coupling constant $\lambda$.

We will now describe the setting for our more general result.

Let $T: \Omega \rightarrow \Omega$ be a homeomorphism of a compact metric space $\Omega$ and let $d \mu$ be a probability measure on $\Omega$ with respect to which $T$ is ergodic. Given a bounded measurable function $f: \Omega \rightarrow \mathbb{R}$, we associate a potential to each $\omega \in \Omega$ by

$$
V_{\omega}(n)=f\left(T^{n}(\omega)\right) \quad \text { for all } n \in \mathbb{Z} .
$$

The corresponding Schrödinger operator is denoted by $H_{\omega}$ :

$$
\left[H_{\omega} \phi\right](n)=\phi(n+1)+\phi(n-1)+V_{\omega}(n) \phi(n), \quad \phi \in \ell^{2}(\mathbb{Z}) .
$$

To describe the requirements we make on $f$ we need to introduce the notion of an essential discontinuity. First, we say that $l \in \mathbb{R}$ is an essential limit of $f$ at $\omega_{0}$ if there exists a sequence $\left\{\Omega_{k}\right\}$ of sets each of positive measure such that for any sequence $\left\{\omega_{k}\right\}$ with $\omega_{k} \in \Omega_{k}$, both $\omega_{k} \rightarrow \omega_{0}$ and $f\left(\omega_{k}\right) \rightarrow l$. If $f$ has more than one essential limit at $\omega_{0}$, we say that $f$ is essentially discontinuous at this point.

Theorem 2. Suppose there is an $\omega_{0} \in \Omega$ such that $f$ is essentially discontinuous at $\omega_{0}$ but is continuous at all points $T^{n} \omega_{0}, n<0$. Then $H_{\omega}$ has no absolutely continuous spectrum for almost every $\omega$.

Remark. Last and Simon [13, $\S 5-6]$ proved the following: if a potential $V$ is the limit (in the Tychonoff topology) of a sequence of translates of a second potential, $W$, then the absolutely continuous spectrum of the operator with potential $V$ contains that of the operator with potential $W$. This theorem permits one to extend the absence of absolutely continuous spectrum from almost every $\omega$ to every $\omega$ in some circumstances. We will use this result in the proof of Theorem 1.

Our strategy will be to revisit a result of Kotani [11]: Given an ergodic family of aperiodic potentials that take only finitely many values, the resulting Schrödinger operators have no absolutely continuous spectrum. The proof of this result rests on an important consequence of Kotani theory $[9,10,11,15]$. In the presence of absolutely continuous spectrum, the potentials are deterministic in the sense that they are uniquely determined by their values on a half-line (see 
Lemma 2.2). Kotani proved that when $f$ takes only finitely many values, the induced potentials are non-deterministic. We will show that functions $f$ obeying the assumptions of Theorem 2 also give rise to non-deterministic potentials and so deduce the absence of absolutely continuous spectrum for the corresponding operators.

\section{A Short Review of Kotani Theory}

Kotani theory (cf. $[9,10,11,12,15])$ establishes a relation between the absolutely continuous spectrum of an ergodic family of Schrödinger operators and the set of energies at which the Lyapunov exponent vanishes. In this section, we recall some specific results from this theory that are necessary for our proof of Theorem 2. Essentially, we summarize parts of [11].

The setting is as follows: Let $d \nu$ be a measure on the space of potentials $[-\lambda, \lambda]^{\mathbb{Z}}$ for which the shift map $[S V](n)=V(n+1)$ is ergodic. Note that $S$ is a homeomorphism with respect to the Tychonoff topology.

The system $\left([-\lambda, \lambda]^{\mathbb{Z}}, S, d \nu\right)$ gives rise to an ergodic family of Schrödinger operators in $\ell^{2}(\mathbb{Z})$,

$$
[H(V) \phi](n)=\phi(n+1)+\phi(n-1)+V(n) \phi(n), \quad V \in \operatorname{supp}(d \nu) .
$$

For each energy $E$, there is an associated fundamental solution (or transfer matrix):

$$
U(n, E, V)=\left(\begin{array}{cc}
E-V(n) & -1 \\
1 & 0
\end{array}\right) \times \cdots \times\left(\begin{array}{cc}
E-V(1) & -1 \\
1 & 0
\end{array}\right) .
$$

The multiplicative ergodic theorem shows that there is a non-random function $\gamma: \mathbb{R} \rightarrow[0, \infty)$, called the Lyapunov exponent, such that

$$
\lim _{n \rightarrow \infty} \frac{1}{n} \log \|U(n, E, V)\|=\gamma(E)
$$

for $\nu$-a.e. $V \in[-\lambda, \lambda]^{\mathbb{Z}}$. We write $N_{d \nu}=\{E \in \mathbb{R}: \gamma(E)=0\}$ for the set of energies at which the Lyapunov exponent vanishes.

Lemma 2.1 (Kotani; see [11]). If $N_{d \nu}$ has zero Lebesgue measure, then $H(V)$ has no absolutely continuous spectrum for $\nu$-a.e. $V$.

Given a potential $V \in[-\lambda, \lambda]^{\mathbb{Z}}$, we will write $V_{ \pm}$for its restrictions to $\mathbb{Z}_{+}=$ $\{0,1,2, \ldots\}$ and $\mathbb{Z}_{-}=\{\ldots,-2,-1\}$, respectively.

Lemma 2.2 (Kotani; see [11]). If $N_{d \nu}$ has positive Lebesgue measure, then each of $V_{ \pm}$determines $V$ uniquely among potentials in $\operatorname{supp}(d \nu)$.

Remark. Unlike Lemma 2.1, this is not an almost everywhere statement; $V_{-}$ determines $V_{+}$throughout the support of $d \nu$. Indeed, the map from $V_{-}$to $V_{+}$ is continuous in the Tychonoff topology. (This is stated in [11]; for more details see [12, Section 5].) 


\section{Absence of Absolutely Continuous Spectrum}

In this section we will prove Theorem 2 and then use this to prove Theorem 1.

Let us fix $\lambda>0$ once and for all such that $[-\lambda, \lambda]$ contains the range of $f$. As in the introduction, we associate a potential $V_{\omega} \in[-\lambda, \lambda]^{\mathbb{Z}}$ to each $\omega \in \Omega$ by $V_{\omega}(n)=f\left(T^{n}(\omega)\right)$. We write $d \nu$ for the measure on $[-\lambda, \lambda]^{\mathbb{Z}}$ induced by the measure $d \mu$ on $\Omega$.

When $f$ is continuous, the map $\omega \mapsto V_{\omega}$ is also continuous and so takes $\operatorname{supp}(d \mu)$ onto $\operatorname{supp}(d \nu)$. (Under a continuous map, the image of a compact set is compact, while the inverse image of a closed set is closed.) When $f$ is not continuous, it is possible for the support of $d \nu$ to contain potentials that do not correspond to any point $\omega \in \Omega$. This plays an important role in the proof of Theorem 2 which we will now present.

Proof of Theorem 2. We will show that there are potentials in $\operatorname{supp}(d \nu)$ which agree on $\mathbb{Z}_{-}$but take different values at $n=0$. By Lemma 2.2 this implies that $N_{d \nu}$ has zero Lebesgue measure. Absence of absolutely continuous spectrum for $\nu$-a.e. potential then follows by Lemma 2.1.

Let $l$ be an essential limit of $f$ at $\omega_{0}$. As $f$ is essentially discontinuous at $\omega_{0}$, it suffices to construct a potential in $\operatorname{supp}(d \nu)$ that agrees with $V_{\omega_{0}}$ on $\mathbb{Z}_{-}$and takes the value $l$ at $n=0$.

Let $\left\{\Omega_{k}\right\}$ be a sequence of sets which exhibits the fact that $l$ is an essential limit of $f$. Since each has positive $\mu$-measure, we can find points $\omega_{k} \in \Omega_{k}$ so that $V_{\omega_{k}}$ is in the support of $d \nu$; indeed, this is the case for almost every point in $\Omega_{k}$.

As $\omega_{k} \rightarrow \omega_{0}$ and $f$ is continuous at each of the points $T^{n} \omega_{0}, n<0$, it follows that $V_{\omega_{k}}(n) \rightarrow V_{\omega_{0}}(n)$ for each $n<0$. Moreover, since $f\left(\omega_{k}\right)$ converges to $l$, we also have $V_{\omega_{k}}(0) \rightarrow l$. We can guarantee convergence of $V_{\omega_{k}}(n)$ for $n>0$ by passing to a subsequence because $[-\lambda, \lambda]^{\mathbb{Z}}$ is compact. Let us denote this limit potential by $V$.

As each $V_{\omega_{k}}$ lies in $\operatorname{supp}(d \nu)$, so does $V$; moreover, $V(0)=l$ and $V(n)=$ $V_{\omega_{0}}(n)$ for each $n<0$. The construction of this potential completes the proof of the theorem for reasons set forth in the opening paragraphs.

Proof of Theorem 1. This result fits into the framework of Theorem 2, where $\Omega=\mathbb{R} / \mathbb{Z}, d \mu$ is Lebesgue measure on the torus $\mathbb{R} / \mathbb{Z}$, and $T$ is the rotation by $\alpha$, that is, $T(\omega)=\omega+\alpha \bmod 1$. Lastly, we write $\omega_{0}$ for the point at which $f$ is discontinuous.

Let us say that $l$ is a limiting value of $f$ at $\omega_{0}$ if there is a sequence $\left\{\omega_{k}\right\}$ in $\Omega \backslash\left\{\omega_{0}\right\}$ such that $\omega_{k} \rightarrow \omega_{0}$ and $f\left(\omega_{k}\right) \rightarrow l$. As $f$ has a non-removable discontinuity at $\omega_{0}$, it has more than one limiting value at this point. Moreover, since $f$ is continuous away from $\omega_{0}$, any limiting value is also an essential limitsimply choose each $\Omega_{k}$ to be a suitably small interval around $\omega_{k}$. 
This shows that $f$ has an essential discontinuity at $\omega_{0}$. As the orbit of $\omega_{0}$ never returns to this point, $f$ is continuous at each point $T^{n} \omega_{0}, n \neq 0$. Therefore, Theorem 2 is applicable and shows that $H_{\omega}$ has no absolutely continuous spectrum for Lebesgue-a.e. $\omega \in[0,1)$.

It remains to show that the absolutely continuous spectrum of $H_{\omega}$ is empty for all $\omega \in \mathbb{R} / \mathbb{Z}$. We begin by fixing $\omega_{1}$ such that $H_{\omega_{1}}$ has no absolutely continuous spectrum and such that the orbit of $\omega_{1}$ does not meet $\omega_{0}$; almost all $\omega_{1}$ have these properties.

Given an arbitrary $\omega \in \mathbb{R} / \mathbb{Z}$, we may choose a sequence of integers $\left\{n_{i}\right\}$ so that $T^{n_{i}}(\omega) \rightarrow \omega_{1}$. As $f$ is continuous on the orbit of $\omega_{1}$, the potentials associated to $T^{n_{i}}(\omega)$ converge pointwise to $V_{\omega_{1}}$. By the result of Last and Simon described in the introduction, the absolutely continuous spectrum cannot shrink under pointwise approximation using translates of a single potential. Thus, the operator with potential $V_{\omega}$ cannot have absolutely continuous spectrum. This concludes the proof.

Remark. The proof of Theorem 1 shows that for functions $f$ on the torus, every isolated (non-removable) discontinuity is essential. As a result, the arguments presented extend to show absence of absolutely continuous spectrum for all $\theta$ when $f$ has finitely many discontinuities. If $f$ has infinitely many discontinuities, these methods still work in most cases; Theorem 2 describes in detail what is needed from $f$.

\section{Acknowledgment}

We thank Barry Simon for valuable comments.

\section{References}

[1] S. Aubry, Metal-insulator transition in one-dimensional deformable lattices, Bifurcation Phenomena in Mathematical Physics and Related Topics, Eds. C. Bardos and D. Bessis, D. Reidel Publisher Co., Boston, Dordrecht, London (1980), 163-184

[2] K. Bjerklöv, Positive Lyapunov exponent for a class of 1-d quasi-periodic Schrödinger equations - the discrete case, Preprint (2003)

[3] J. Bourgain, Green's function estimates for lattice Schrödinger operators and applications. Annals of Mathematics Studies, 158. Princeton University Press, Princeton, NJ, 2005.

[4] J. Bourgain and M. Goldstein, On nonperturbative localization with quasi-periodic potential, Ann. of Math. 152 (2000), 835-879

[5] J. Bourgain and S. Jitomirskaya, Absolutely continuous spectrum for $1 D$ quasiperiodic operators, Invent. Math. 148 (2002), 453-463

[6] F. Delyon and D. Petritis, Absence of localization in a class of Schrödinger operators with quasiperiodic potential, Commun. Math. Phys. 103 (1986), 441-444

[7] S. Jitomirskaya, Metal-insulator transition for the almost Mathieu operator, Ann. of Math. 150 (1999), 1159-1175

[8] S. Klein, Anderson localization for the discrete one-dimensional quasi-periodic Schrödinger operator with potential defined by a Gevrey-class function. J. Funct. Anal. 218 (2005), 255-292. 
[9] S. Kotani, Ljapunov indices determine absolutely continuous spectra of stationary random one-dimensional Schrödinger operators, Stochastic analysis (Katata/Kyoto, 1982), 225247, North-Holland Math. Library, 32, North-Holland, Amsterdam, 1984

[10] - One-dimensional random Schrödinger operators and Herglotz functions, Probabilistic methods in mathematical physics (Katata/Kyoto, 1985), 219-250, Academic Press, Boston, MA, 1987

[11] _ Jacobi matrices with random potentials taking finitely many values, Rev. Math. Phys. 1 (1989), 129-133

[12] Generalized Floquet theory for stationary Schrödinger operators in one dimension, Chaos Solitons Fractals 8 (1997), 1817-1854

[13] Y. Last and B. Simon, Eigenfunctions, transfer matrices, and absolutely continuous spectrum of one-dimensional Schrödinger operators, Invent. Math. 135 (1999), 329-367

[14] V. A. Mandel'shtam and S. Ya. Zhitomirskaya, 1D-quasiperiodic operators. Latent symmetries, Commun. Math. Phys. 139 (1991), 589-604

[15] B. Simon, Kotani theory for one-dimensional stochastic Jacobi matrices, Commun. Math. Phys. 89 (1983), 227-234

Mathematics 253-37, California Institute of Technology, Pasadena, CA 91125, USA

E-mail address: damanik@its.caltech.edu

Department of Mathematics, University of California, Los Angeles, CA 90055 , USA

E-mail address: killip@math.ucla.edu 